\title{
Notification that New Names and New Combinations Have Appeared in Volume 44, No. 3, of the IJSB
}

This listing of names published in a previous issue of IJSB is provided as a service to bacteriology to assist in the recognition of new names and new descriptions. This procedure was proposed by the Judicial Commission [Minute II (ii), Int. J. Syst. Bacteriol. 41:185, 1991]. The names given herein have priority according to the issue of the IJSB in which they were published.

\begin{tabular}{|c|c|c|c|}
\hline Name & Proposed as: & Authors & $\begin{array}{l}\text { IJSB reference } \\
\text { description: }\end{array}$ \\
\hline Pseudomonas flavescens & sp. nov. & Hildebrand et al. & 44(3):413 \\
\hline Roseococcus & gen. nov. & Yurkov et al. & $44(3): 430$ \\
\hline Roseococcus thiosulfatophilus & sp. nov. & Yurkov et al. & $44(3): 432$ \\
\hline Erythromicrobium & gen. nov. & Yurkov et al. & 44(3):432 \\
\hline Erythromicrobium ramosum & sp. nov. & Yurkov et al. & $44(3): 432$ \\
\hline Erythrobacter litoralis & sp. nov. & Yurkov et al. & 44(3):432 \\
\hline Lactobacillus kefirgranum & sp. nov. & Takizawa et al. & $44(3): 438$ \\
\hline Lactobacillus parakefir & sp. nov. & Takizawa et al. & 44(3):439 \\
\hline Flavobacterium scophthalmum & sp. nov. & Mudarris et al. & 44(3):450 \\
\hline Staphylococcus vitulus & sp. nov. & Webster et al. & 44(3):459 \\
\hline Nocardiopsis halophila & sp. nov. & Al-tai and Ruan & 44(3):477 \\
\hline Mycoplasma auris & sp. nov. & DaMassa et al. & $44(3): 483$ \\
\hline Mycoplasma cottewii & sp. nov. & DaMassa et al. & 44(3):483 \\
\hline Mycoplasma yeatsii & sp. nov. & DaMassa et al. & 44(3):483 \\
\hline Mycobacterium chlorophenolicum (basonym Rhodococcus chlorophenolicus) & comb. nov. & Häggblom et al. & 44(3):491 \\
\hline Brevundimonas & gen. nov. & Segers et al. & 44(3):507 \\
\hline Brevundimonas diminuta (basonym Pseudomonas diminuta) & comb. nov. & Segers et al. & 44(3):508 \\
\hline Brevundimonas vesicularis (basonym Pseudomonas vesicularis) & comb. nov. & Segers et al. & 44(3):508 \\
\hline Rhizobium ciceri & sp. nov. & Nour et al. & $44(3): 520$ \\
\hline Nocardioides plantarum & sp. nov. & Collins et al. & 44(3):525 \\
\hline Halothermothrix & gen. nov. & Cayol et al. & 44(3):538 \\
\hline Halothermothrix orenii & sp. nov. & Cayol et al. & 44(3):538 \\
\hline Helicobacter pametensis & sp. nov. & Dewhirst et al. & 44(3):559 \\
\hline Haloanaerobium salsugo & sp. nov. & Bhupathiraju et al. & 44(3):570 \\
\hline Methanolobus taylori & sp. nov. & Oremland and Boone & 44(3):574 \\
\hline Acidaminococcus fermentans & emend. & Cook et al. & 44(3):577 \\
\hline Propioniferax & gen. nov. & Yokota et al. & 44(3):581 \\
\hline Propioniferax innocua (basonym Propionibacterium innocuum) & comb. nov. & Yokota et al. & 44(3):581 \\
\hline Enterobacter cancerogenus pro synon. Enterobacter taylorae & synon. & Schønheyder et al. & 44(3):586 \\
\hline
\end{tabular}

